\title{
Quality Assessment and Ecotype Distinction for Panax quinquefolius L. from China and Canada by ${ }^{1} \mathrm{H}$ NMR and Chemometrics
}

\author{
Caimei Gu, Zenghui Wang, Labin Wu, Linfang Huang* \\ Institute of Medicinal Plant Development, Chinese Academy of Medical Sciences and Peking Union Medical College, Beijing, China.
}

\section{ARTICLE INFO}

Article history:

Received on: 09/11/2016

Accepted on: 07/01/2017

Available online: 30/05/2017

Key words:

Ecotype, Quality control, $P$. quinquefolius,

Chemometrics, ${ }^{1} \mathrm{H}$ NMR.

\begin{abstract}
Panax quinquefolius $\mathrm{L}$. is one of the most widely consumed and cultivated herbal medicines around the world. China and Canada are the two major producing countries. However, research on the ecotype distinction of $P$. quinquefolius from Canada has never been reported, and the quality evaluation of $P$. quinquefolius between China and Canada using nuclear magnetic resonance $\left({ }^{1} \mathrm{H}-\mathrm{NMR}\right)$ is limited. Here, we investigated the ${ }^{1} \mathrm{H}$ NMR key signals and ecological factors of $P$. quinquefolius samples, and the data were further analyzed by principal component analysis (PCA) and partial least squares discriminant analysis (PLS-DA). The key signals were identified as sugar and methyl, which distinguished all the samples into two different ecotypes. This is the first report of ecotype division for $P$. quinquefolium worldwide. The results showed quality variation of $P$. quinquefolium from different geographic areas, implying the ecological adaptation and biodiversity. Our findings also demonstrate the critical need for improving quality and quality standardization, appropriate ecological regionalization and promoting industrialized development of $P$. quinquefolium.
\end{abstract}

\section{INTRODUCTION}

Panax quinquefolius L. is one of the most important herbs in the world, and is native to North America in USA and Canada; it is now widely cultured in many parts all over the world, including China (Christensen et al., 2006). The history of $P$. quinquefolius, in China, was first recorded in Ben Cao Gang $\mathrm{Mu}$ in the Ming Dynasty (Li, 2004). Presently, the cultivation of the herb has been extended on a large scale in Jilin and Shandong provinces. Canada, China and USA are the three major producing areas. China has gradually become the major production, consumption and export country instead of traditional import country.

It was reported that the contents and composition of perceived pharmacological properties varied significantly among populations (Li et al., 1996; Tang et al., 2016; Qi et al., 2016). The pharmacological effects of ginseng roots has been attributed primarily to ginsenosides, a triterpenoid saponin glycoside.

\footnotetext{
* Corresponding Author

Email: lfhuang @implad.ac.cn
}

Ginsenosides were recorded as indicator compound in the Chinese Pharmacopoeia and the United States Pharmacopeia. The grading and pricing of $P$. quinquefolius are primarily determined by the ginsenosides of harvested roots (Lim et al., 2005). The ecotype is a population concept put forward by Turesson in 1921, and Odum improved it (Liu et al., 2004; Odum, 1996). The ecotype of $P$. quinquefolius from China has been investigated in our lab, which showed that two chemoecotypes of $P$. quinquefolius in China, ginsenosides Rb1-Re from outside Great Wall and Rg2$\mathrm{Rd}$ from inside Great Wall with distinct climatic characteristics (Wang and Huang, 2015; Wang et al., 2015; Huang et al., 2013). To date, some studies have been reported for quality assessment of P. quinquefolius (Ludwiczuk et al., 2005; Xu et al., 2011; Chan et al., 2000; Sun et al., 2012; Zhao et al., 2015; Yang et al., 2012), but the ecotype of $P$. quinquefolius from major productive country Canada has never been conducted. The current article investigates the differences in quality and ecotype of $P$. quinquefolius from Canada and China by ${ }^{1} \mathrm{H}-\mathrm{NMR}$ spectroscopy coupled with chemometrics, which includes PCA and PLS-DA. This paper aims to establish an effective and rapid metabolomics method for geographical origin traceability of $P$. quinquefolius in the world. 


\section{MATERIALS AND METHODS}

\section{Sample collection}

The samples of $P$. quinquefolius were collected from 6 locations, which include China and Canada populations (Table 1). Three independent plants were collected from each location. Professor Huang Linfang identified the botanical specimens, and the voucher specimens were deposited in the Institute of Medicinal Plant Development, Chinese Academy of Medical Sciences, Beijing, China. The typical root and aerial part of $P$. quinquefolius is shown in Fig.1.

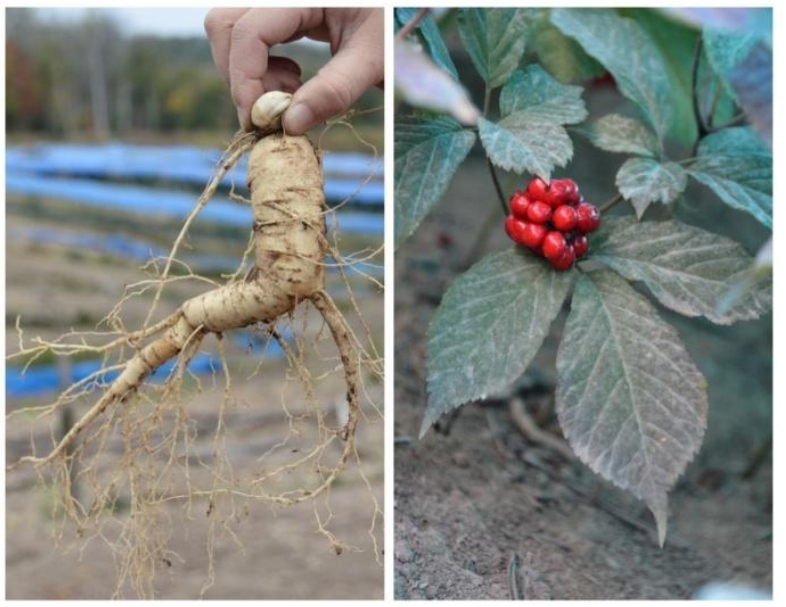

Fig.1: The root and aerial part of $P$. quinquefolius.

\section{Chemicals and reagents}

For ${ }^{1} \mathrm{H}$ NMR analysis, methanol-d4 ( $\left.\mathrm{CD}_{3} \mathrm{OD}, 99.8 \%\right)$, dimethyl sulfoxide-d6 (DMSO-d6, 99.9\%), and deuterated pyridine $\left(\mathrm{C}_{5} \mathrm{D}_{5} \mathrm{~N}, 99.5 \%\right)$ were purchased from Cambridge Isotope
Laboratories, Inc. (Miami, FL, USA). The $P$. quinquefolius samples, labeled as CSDa1-3, CSDb1-3, CJLa1-3, CJLb1-3, were collected from China;samplesCANa1-3 and CANb1-3, with origins in Canada, were purchased from Hong Kong Lin Shi Ginseng Antler Co., Ltd. The detailed information of the plant materials in this study is shown in Table 1.

\section{Preparation of samples}

$\mathrm{CD}_{3} \mathrm{OD}$, DMSO-d6 and $\mathrm{C}_{5} \mathrm{D}_{5} \mathrm{~N}$ were tested to dissolve the lyophilized samples; $\mathrm{CD}_{3} \mathrm{OD}$ was found the best solvent. $30 \mathrm{mg}$ of each samplewas extracted with $1 \mathrm{ml}$ of $\mathrm{CD}_{3} \mathrm{OD}$. The extractions were vortexed vigorously for $30 \mathrm{~s}$, and then were sonicated for 40 min at room temperature. NMR samples were prepared by shaking manually, sonicated the insoluble material at $2000 \mathrm{~g}$ for a further 5 min, and adjusted to $\mathrm{pH} 7.0 \pm 0.003$ using deuterated base and/or acid. Finally, it wastransferring the supernatant of the extracts into $5 \mathrm{~mm}$ NMR tubes for NMR analysis (Shin et al., 2007).

\section{${ }^{1}$ H NMR analysis}

One-dimensional ${ }^{1} \mathrm{H}$ NMR spectra were measured at a temperature of $300 \mathrm{Kon}$ a $600.13 \mathrm{MHz}$ BrukerAvance spectrometer (BrukerAnalytische $\mathrm{GmbH}$, Rheinstetten, Germany) equipped with a broad-band-observe (BBO) probe. A zgcppr pulse sequence wasapplied to suppress the residual water signal. A total of 128transients were collected in $32 \mathrm{~K}$ data points with relaxation delay of 2s. Aspectral width of $9615.4 \mathrm{~Hz}$ and an acquisition time per scan of 1.70 s were used.

Prior to Fourier transformation, an exponential line broadening function of $0.30 \mathrm{~Hz}$ was applied to the free induction decay. The chemical shifts for all samples were referenced to TMS (tetramethylsilane) at $0.00 \mathrm{ppm}$.

Table 1: P. quinquefolius samples collected from different locations

\begin{tabular}{|c|c|c|c|c|c|c|}
\hline Number & Lat/Lon & Location & Date & Voucher numbers & State & Years-grow \\
\hline CSDa1 & $\mathrm{N} 37.13^{\circ} \mathrm{E} 122.13^{\circ}$ & Kouzili, Shandong, China & 2015.10 & Implad2015ZYH-CSDa1 & Fresh & 4 \\
\hline CSDa2 & $\mathrm{N} 37.13^{\circ} \mathrm{E} 122.13^{\circ}$ & Kouzili, Shandong, China & 2015.10 & Implad2015ZYH- CSDa2 & Fresh & 4 \\
\hline CSDa3 & $\mathrm{N} 37.13^{\circ} \mathrm{E} 122.13^{\circ}$ & Kouzili, Shandong, China & 2015.10 & Implad2015ZYH- CSDa3 & Fresh & 4 \\
\hline CSDb1 & $\mathrm{N} 37.4^{\circ} \mathrm{E} 121.95^{\circ}$ & Dashuipo, Shandong, China & 2015.10 & Implad2015ZYH- CSDb1 & Fresh & 4 \\
\hline $\mathrm{CSDb} 2$ & $\mathrm{~N} 37.4^{\circ} \mathrm{E} 121.95^{\circ}$ & Dashuipo, Shandong, China & 2015.10 & Implad2015ZYH- CSDb2 & Fresh & 4 \\
\hline CSDb3 & $\mathrm{N} 37.4^{\circ} \mathrm{E} 121.95^{\circ}$ & Dashuipo, Shandong, China & 2015.10 & Implad2015ZYH- CSDb3 & Fresh & 4 \\
\hline CJLa1 & $\mathrm{N} 42.33^{\circ} \mathrm{E} 127.3^{\circ}$ & Fusong, Jilin, China & 2015.10 & Implad2015ZYH- CJLa1 & Fresh & 4 \\
\hline CJLa2 & $\mathrm{N} 42.33^{\circ} \mathrm{E} 127.3^{\circ}$ & Fusong, Jilin, China & 2015.10 & Implad2015ZYH- CJLa2 & Fresh & 4 \\
\hline CJLa3 & $\mathrm{N} 42.33^{\circ} \mathrm{E} 127.3^{\circ}$ & Fusong, Jilin, China & 2015.10 & Implad2015ZYH- CJLa3 & Fresh & 4 \\
\hline CJLb1 & $\mathrm{N} 41.44^{\circ} \mathrm{E} 125.97^{\circ}$ & Tonghua, Jilin, China & 2015.10 & Implad2015ZYH- CJLb1 & Fresh & 4 \\
\hline CJLb2 & $\mathrm{N} 41.44^{\circ} \mathrm{E} 125.97^{\circ}$ & Tonghua, Jilin, China & 2015.10 & Implad2015ZYH- CJLb2 & Fresh & 4 \\
\hline CJLb3 & $\mathrm{N} 41.44^{\circ} \mathrm{E} 125.97^{\circ}$ & Tonghua, Jilin, China & 2015.10 & Implad2015ZYH-CJLb3 & Fresh & 4 \\
\hline CANa1 & $\mathrm{N} 43.98^{\circ} \mathrm{W} 79.19^{\circ}$ & Toronto Canada & 2015.10 & Implad2015ZYH- CANa1 & Dry & 4 \\
\hline $\mathrm{CANa} 2$ & $\mathrm{~N} 43.98^{\circ} \mathrm{W} 79.19^{\circ}$ & Toronto Canada & 2015.10 & Implad2015ZYH- CANa2 & Dry & 4 \\
\hline CANa3 & $\mathrm{N} 43.98^{\circ} \mathrm{W} 79.19^{\circ}$ & Toronto Canada & 2015.10 & Implad2015ZYH- CANa3 & Dry & 4 \\
\hline $\mathrm{CANb} 1$ & $\mathrm{~N} 49.279^{\circ} \mathrm{W} 123.05^{\circ}$ & Vancouver Canada & 2015.10 & Implad2015ZYH- CANb1 & Dry & 4 \\
\hline $\mathrm{CANb} 2$ & $\mathrm{~N} 49.279^{\circ} \mathrm{W} 123.05^{\circ}$ & Vancouver Canada & 2015.10 & Implad2015ZYH- CANb2 & Dry & 4 \\
\hline CANb3 & $\mathrm{N} 49.279^{\circ} \mathrm{W} 123.05^{\circ}$ & Vancouver Canada & 2015.10 & Implad2015ZYH- CANb3 & Dry & 4 \\
\hline
\end{tabular}


Table 2: The ecological factors of sampling locations.

\begin{tabular}{|c|c|c|c|c|c|c|c|c|}
\hline \multirow[b]{2}{*}{ Location } & \multicolumn{8}{|c|}{ Major ecological factors } \\
\hline & $\begin{array}{l}\text { Annual air } \\
\text { temperature }\end{array}$ & $\begin{array}{l}\text { Relative } \\
\text { humidity }\end{array}$ & $\begin{array}{c}\text { Annual } \\
\text { precipitation }\end{array}$ & $\begin{array}{c}\text { Annual } \\
\text { solar insulation }\end{array}$ & $\begin{array}{c}\text { Average air } \\
\text { temperature in } \\
\text { January }\end{array}$ & $\begin{array}{c}\text { Minimum air } \\
\text { temperature in } \\
\text { January }\end{array}$ & $\begin{array}{c}\text { Average air } \\
\text { temperature in } \\
\text { July }\end{array}$ & $\begin{array}{c}\text { Maximum air } \\
\text { temperature in } \\
\text { July }\end{array}$ \\
\hline CSD1 & 14 & 70.2 & 723.6 & 1582.2 & 6.37 & 6.33 & 22.8 & 22.8 \\
\hline CSD2 & 13.1 & 70.2 & 678.9 & 1573.76 & 2.54 & 1.09 & 23.7 & 25.9 \\
\hline CSD3 & 13.9 & 71.3 & 708.1 & 1543.95 & 3.69 & 2.22 & 24 & 26.2 \\
\hline CJL1 & 6.03 & 64.8 & 831.6 & 1431.9 & -14.3 & -21 & 21.3 & 27.3 \\
\hline CJL2 & 3.17 & 68.7 & 759.6 & 1426.5 & -18.8 & -26.4 & 20 & 26.5 \\
\hline CJL3 & 4.34 & 68.9 & 861.4 & 1435,36 & -16.7 & -24.1 & 20.3 & 26.5 \\
\hline CAN1 & 6.27 & 71.2 & 1237.35 & 1240.8 & -2.25 & -5.9 & 16.6 & 26.3 \\
\hline CAN2 & 7.35 & 71 & 964.8 & 1291.5 & -7.66 & -12.3 & 20.8 & 29 \\
\hline CAN3 & 5.21 & 71.3 & 1047.55 & 1258.03 & -4.05 & -8 & 16.3 & 26.2 \\
\hline
\end{tabular}

CSD1-3 and CJL1 are Chinese samples data; CAN1-3 are the Canadian samples data.

\section{Ecological factors}

The data of ecological factors were collected from the NASA Atmospheric Science Data Center, which covered the whole growth process of $P$. quinquefolius from the year 2011 to 2015. The average values of ecological factors listed in Table 2, including annual air temperature, relative humidity, annual precipitation, annual average solar isolation, average air temperature in January, and minimum air temperature in January, average air temperature in July, and maximum air temperature in July, at 6 locations.

\section{Data analysis}

The NMR spectra of all the $P$. quinquefolius samples were bucketed in $0.04 \mathrm{ppm}$. Bucketing of spectra was performed using MestReNova (version 6.0.4.SantiagodeCompostela, Spain: Mestrelab Research) software and Chenomx NMR suite software (version5.1. Alberta, Canada: Chenomx Inc.). The resulting data were converted to Microsoft Office Excel (version 2007. WA, USA: Microsoft) format, and imported into SIMCA P+ software (version 12.0.Umea, Sweden, Umetrics)(Park et al., 2013). The method of mean-centered and scaling with Pareto (Par) scaling, were used in PLS-DA.

\section{RESULTS AND DISCUSSION}

\section{${ }^{1}$ H-NMR spectra profiling}

To obtain the best separation for all the integrated signals in ${ }^{1} \mathrm{H}$ NMR spectra, NMR solvents were optimized in this study. Based on the dissolvability of the main pharmacological components, three solvent systems involving $\mathrm{CD}_{3} \mathrm{OD}$, DMSO-d6 and $\mathrm{C}_{5} \mathrm{D}_{5} \mathrm{~N}$ were investigated. $\mathrm{CD}_{3} \mathrm{OD}$ showed better separation for the signals of the analyzed ginsenosides, and it was the preferred ${ }^{1} \mathrm{H}$ NMR solvent finally. In Fig. 2I, examples of NMR spectra of P. quinquefolius are shown. Differences can be observed from the spectra, PCA and PLS-DA was utilized to further analyze the differences in the spectra of the samples.

\section{Chemometric analysis}

PCA was performed on the pretreated NMR spectra of all the studied $P$. quinquefolius samples. Fig. 3A presents the differences between samples from China and Canada. The samples from Jilin in China and the samples from Canada clustered into one group, while samples from Shandong in China were in a distinct group, which is similar result with our previous research(Huang et al., 2013). In order to further analyze the spectra of different samples, 16 main peaks were selected based on their intensity data.

These data were then imported into Excel to generate a curve graph (Fig. 3B). The figure shows the differences in content of the main compounds in various samples. It is interesting to note that the peaks in the range of $3.0 \mathrm{ppm}$ to $5.5 \mathrm{ppm}$ (sugar region) and those in the range of $0.5 \mathrm{ppm}$ to $2.0 \mathrm{ppm}$ (aliphatic region) show an opposite trend (Shin et al., 2007; Lee et al., 2009; Jocham et al., 2007). The peaks in the range of $0.5 \mathrm{ppm}$ to $2.0 \mathrm{ppm}$ and 3.0 ppm to $5.5 \mathrm{ppm}$ are pointing to opposite directions, meaning that if higher $0.5 \mathrm{ppm}$ to $2.0 \mathrm{ppm}$ peaks are present, then lower $3.0 \mathrm{ppm}$ to $5.5 \mathrm{ppm}$ peaks are present, and vice versa.

This result shows that location may influence the quality of $P$. quinquefolius significantly. In United States Pharmacopeia USP35, the content of ginsenosides acceptance criteria is no less than $4.0 \%$ of total ginsenosides $\left(\mathrm{Rg}_{1}, \mathrm{Re}, \mathrm{Rb}_{1}, \mathrm{Rc}, \mathrm{Rb}_{2}\right.$ and $\left.\mathrm{Rd}\right)$ on the dried basis, while in Chinese Pharmacopoeia 2015, the acceptance criteria is no less than $2.0 \%$ of total ginsenosides $\left(\operatorname{Rg}_{1}\right.$ $\operatorname{Re}$ and $\mathrm{Rb}_{1}$ ) on the dried basis. The content requirement of ginsenosides is different in the two Pharmacopeias, which illustrate the importance of quality control. 

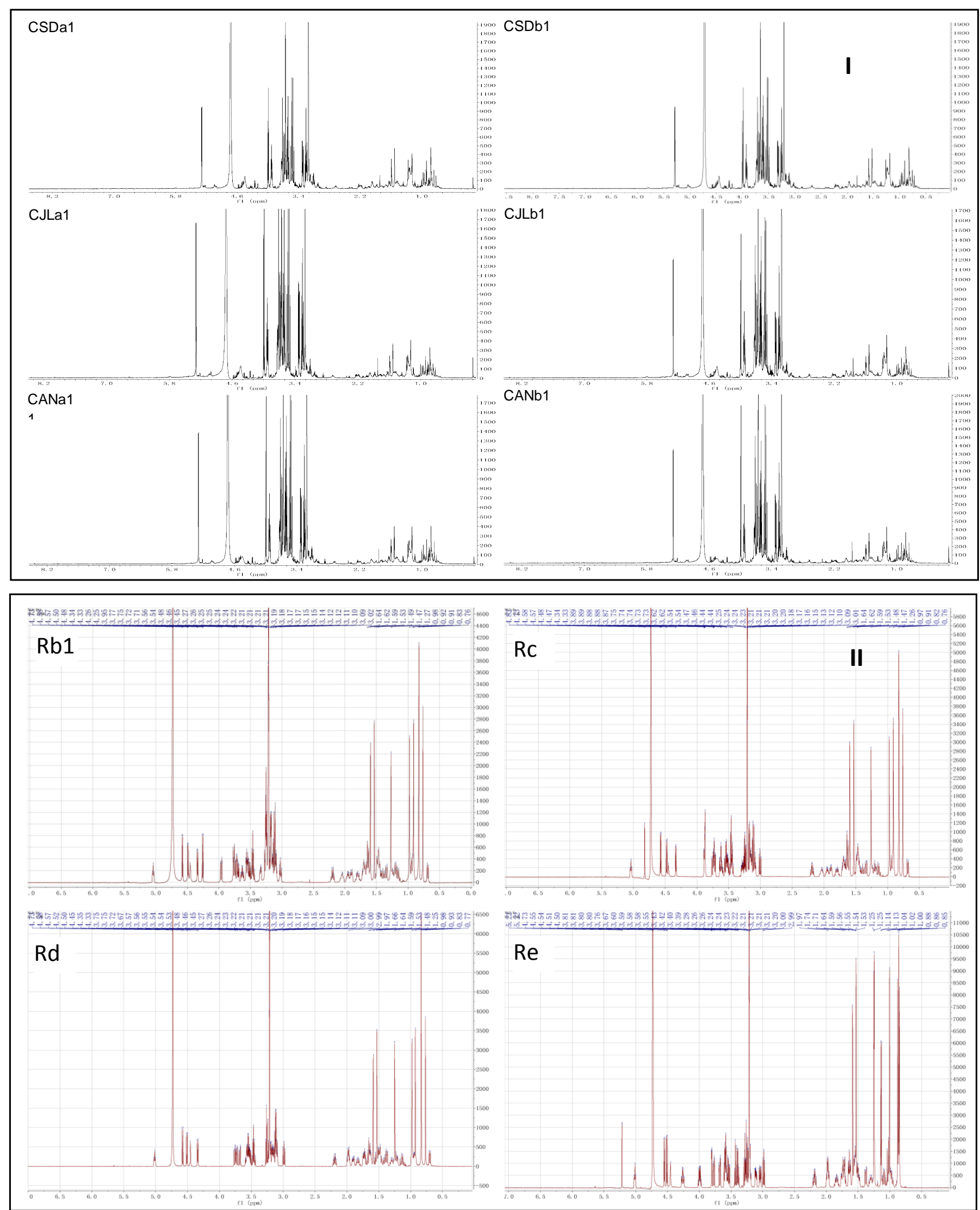

Fig. 2: I Representative ${ }^{1} \mathrm{H}$ NMR spectra of $P$. quinquefoliusfrom China and Canada. II NMR spectra of ginsenoside Rb1, Rc, Rd and Re. 

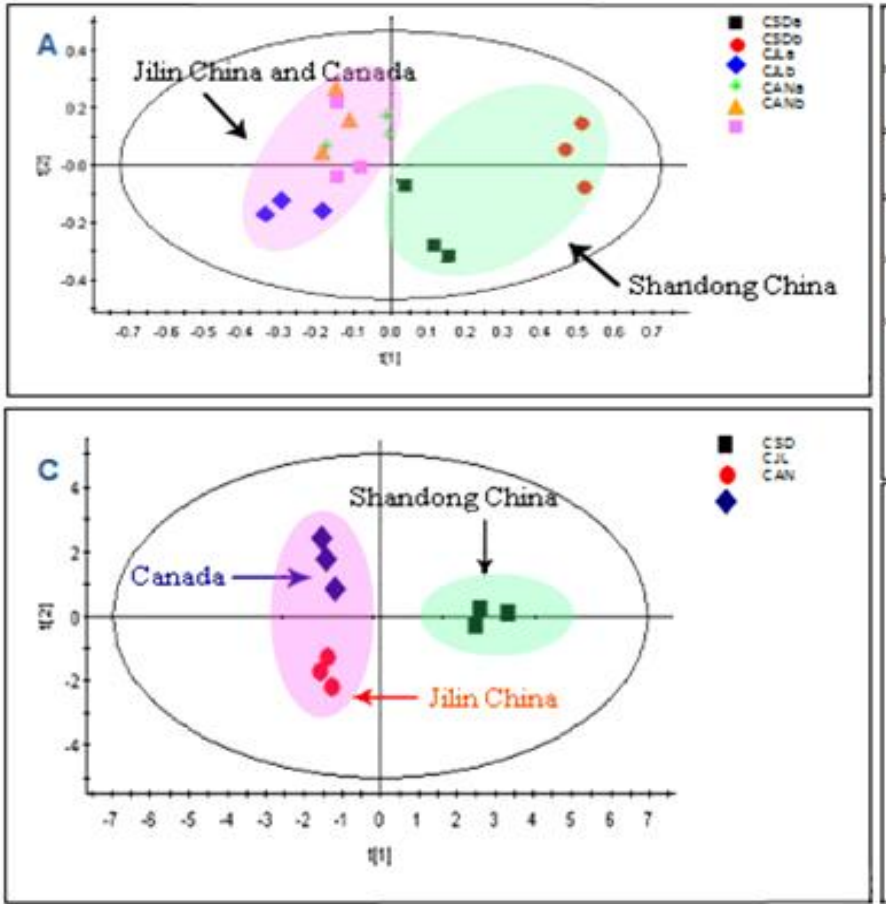

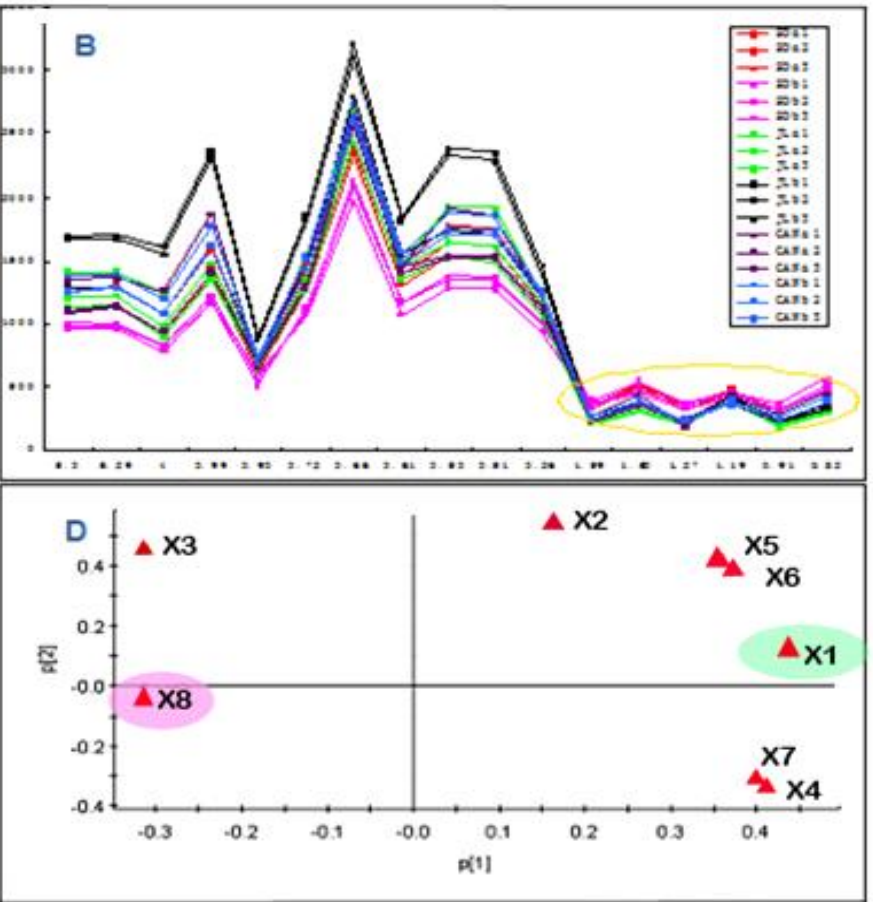

Fig. 3: A The PLS-DA analysis of different $P$. quinquefolius samples $\left(\mathrm{R}^{2} \mathrm{X}[1]=0.400836 \mathrm{R}^{2} \mathrm{X}[2]=0.173504\right)$; B Intensities of selected peaks from 1H NMR profiles of Various $P$. quinquefolius; C The PLS-DA score plots of ecological factors of sampling location $\left(\mathrm{R}^{2} \mathrm{X}[1]=0.570915\right.$ $\mathrm{R}^{2} \mathrm{X}$ [2]=0.299042); D The PLS-DA loading plot of ecological factors of sampling location. X1 Annual air temperature; X2 Relative humidity; X3 Annual precipitation; X4 Annual average solar insolation; X5 Average air temperature in January; X6 Minimum air temperature in January; X7Average air temperature in July; X8 Maximum air temperature in July.

\section{Ecological factors analysis and its effect on phytochemical composition}

Principal component analysis was used to analyze the differences in climate in China (Shandong and Jilin) and Canada. The PLA-DA scores plot shows that ecological factors of Jilin in China and those in Canada clustered into one group, and those in Shandong, China clustered into another group (Fig. 3C). The PCA loadings plot indicated that the annual air temperature and maximum air temperature in July made a great influence on the discrimination of the 2 types of climate (Fig. 3D). Shandong has a relatively higher annual air temperature and relatively lower maximum air temperature in July, which of these above all verified our previous results.

The result was also validated by our previous experiments. In 2008, we reported that the northeast of China including Jilin is the most suitable area for the growth of $P$. quinquefolius, because the climate is very similar to North America (Chen et al., 2008). And in 2011, our data further showed the temperature was the most important environmental factors affecting the content of ginsenosides of $P$. quinquefolius roots (Dong, 2011). Jochum. GM, et al, demonstrated that plants grown, at high temperatures, had less root biomass and greater concentrations of storage root ginsenosides (49\%), than plants grown at low temperatures (Jocham et al., 2007). In the present study, Shandong samples (grown at high environmental temperature) contain higher signal levels in the range of $0.5 \mathrm{ppm}$ to $2.0 \mathrm{ppm}$, and the selected peaks, $1.59,1.53,1.27,1.19,0.91$ $0.82 \mathrm{ppm}$, were mainly correlated to the methyl group of ginsenosides that is referred to in the supplementary information (Fig. 2II). The samples from Jilin and Canada (grown at lower environmental temperature) contain relatively higher levels of signals in the sugar region. We performed discriminatory NMRbased chemical profile studies on $P$. quinquefolius roots from China and Canada. Samples from Shandong, China contain higher content of ginsenosides and relatively lower saccharides than those from Jilin in China and those from Canada. And chemical discrimination was in accordance with their ecological discrimination, and temperature factors were concluded as the most influential ecological variable. All the above results are consistent with our previous work. Consequently, we assume that the ecotype of Canadian P. quinquefolius was outside Great Wall due to the similar latitude to the Northeast of China.

\section{CONCLUSION}

The $P$. quinquefolius are observed with big differences in quality and ecotype between China and Canada, which indicate that the $P$. quinquefolius from Canada is the ecotype of outside Great Wall. This is the first time studied the ecotype distinction of $P$. quinquefolius around the world, and the present approach is 
important and reliable to control the quality and distinguish ecotype.

\section{AUTHORS'CONTRIBUTIONS}

Linfang Huang initiate and all authors designed the study. The sample extraction was conduct by Zenghui Wang and Labin $\mathrm{Wu}$, The method developments were conducted by Caimei $\mathrm{Gu}$ who drafted the manuscript. All authors contributed to the data analyses and to finalizing the manuscript.

\section{CONFLICTS OF INTEREST}

The authors declare that they have no conflict of interest.

\section{ACKNOWLEDGMENTS}

The study was supported by grants from the National Natural Science Foundation of China (No.: 81274013 and $81473315)$

\section{REFERENCES}

ChristensenLP, JensenM, KidmoseU.Simultaneous determination of ginsenosides and polyacetylenes in Panax quinquefolius L. root (Panax quinquefolium L.) by high-performance liquid chromatography. Journal of agricultural and food chemistry. 2006;54(24):8995-9003.

LiSZ. Compendium of Materia Medica (Ben cao Gang mu), Beijing: Foreign Languages Press; 2004.

LiTS,MazzaG, CottrellAC.Ginsenosides in roots and leaves of Panax quinquefolius L.Journal of agricultural and food chemistry. 1996; 44(3):717-720.

Tang X, Gan X T, Rajapurohitam V, et al. North American ginseng (Panax quinquefolius) suppresses $\beta$-adrenergic-dependent signalling, hypertrophy, and cardiac dysfunction. Canadian Journal of Physiology and Pharmacology, 2016, 94(12): 1325-1335.

Qi B, Wang S, Wang Q, et al. Characterization and immunostimulating effects on murine peritoneal macrophages of a novel protein isolated from Panax quinquefolius L. Journal of Ethnopharmacology, 2016, 193: 700-705.

Lim W, MudgeKW, Vermeylen F.Effects of population, age, and cultivation methods on ginsenoside content of wild Panax quinquefolius L.Journal of agricultural and food chemistry. 2005; 53(22): 8498-8505.

LiuZY, ChenBG, Xie ZS.Advances in plant ecotype classification. Ecologic Science. 2004; 23(4): 365-369.

Odum EP. Fundamentals of Ecology Saunders: Philadelphia; 1997.

WangZH, HuangLF.Panax quinquefolius: An overview of the contaminants. Phytochemistry Letters. 2015; 11: 89-94.

WangYPet al. Chemical analysis of Panax quinquefolius (North American ginseng): A review. Journal of Chromatography A. 2015; 1426: $1-15$.

Huang LF, et al.Quality variation and ecotype division of Panax quinquefolium in China.Acta Pharmaceutica Sinica. 2013; 48(4): 580-589.
Ludwiczuk A, Nyiredy S, Wolski T.Separation of the ginsenosides fraction obtained from the roots of Panax quinquefolius $\mathrm{L}$. cultivated in Poland. Journal of Planar Chromatography-Modern TLC. 2005; 18(102): 104-107.

XuY,LiuY,JieS.Quality Assessment of Saponins from Aerial Parts of Panax quinquefolius L. by LC-ELSD Fingerprints and LC Quantitative Analysis. Planta Medica. 2011;77: 94.

ChanTWD et al.Differentiation and authentication of Panax ginseng, Panax quinquefolius. and ginseng products by using HPLC/MS. Analytical chemistry. 2000;72(6): 1281-1287.

Sun BS, Xu MY, Li Z.UPLC-Q-TOF-MS/MS analysis for steaming times-dependent profiling of steamed Panax quinquefolius L. and its ginsenosides transformations induced by repetitious steaming.Journal of ginseng research. 2012;36(3): 277-290.

Zhao HY et al. Metabolomic quality control of commercial Asian ginseng, and cultivated and wild Panax quinquefolius $\mathrm{L}$. using ${ }^{1} \mathrm{H}$ NMR and multi-step PCA. Journal of Pharmaceutical and Biomedical Analysis. 2015;114: 113-120.

YangSOet al.NMR-based metabolic profiling and differentiation of ginseng roots according to cultivation ages. Journal of Pharmaceutical and Biomedical Analysis. 2012; 58: 19-26.

ShinYS, BangKH,InDS.Fingerprinting analysis of fresh ginseng roots of different ages using1H-NMR spectroscopy and principal components analysis.Archives of pharmacal research. 2007;30(12):16251628 .

ParkSJ, Hyun SH,SuhHW.Biochemical characterization of cultivated Cordycepsbassiana mycelia and fruiting bodies by ${ }^{1} \mathrm{H}$ nuclear magnetic resonance spectroscopy.Metabolomics. 2013;9(1):236-246.

Kang J, Lee S, Kang S.NMR-based metabolomics approach for the differentiation of ginseng (Panax ginseng) roots from different origins.Archives of pharmacal research. 2008;31(3): 330-336.

Lee EJ, Shaykhutdinov R, Weljie AM.Quality assessment of ginseng by ${ }^{1} \mathrm{H}$ NMR metabolite fingerprinting and profiling analysis.Journal of agricultural and food chemistry. 2009;57(16): 75137522

Chen SL, Zhou YQ, Xie CX. Suitability evaluation of Panax quinquefolius L.'s producing area based on TCMGIS-1. China Journal of Chinese Materia Medica. 2008; 33(7): 741-745.

Dong L. Study on the correlations between the quality characteristics of Panax ginseng, Panax quinquefolius L. and ecological factors. Zhengzhou: Henan University of Traditional Chinese Medicine; 2011.

Jochum GM, Mudge KW, Thomas RB.Elevated temperatures increase leaf senescence and root secondary metabolite concentrations in the understory herb Panax quinquefolius L. (Araliaceae). American journal of botany. 2007;94(5): 819-826.

\section{How to cite this article:}

Gu C, Wang Z, Wu L, Huang L. Quality Assessment and Ecotype Distinction for Panax quinquefolius L. from China and Canada by ${ }^{1}$ H NMR and Chemometrics. J App Pharm Sci, 2017; 7 (05): 018023. 